W. Hepp (Hrsg.)

Angiologische Notfälle 


\section{$1418817 \%$
011302089}

Anschrift des Herausgebers:

Prof. Dr. med. W. Hepp

Chirurgische Klinik und Poliklinik

Universitätsklinikum Rudolf Virchow

Standort Charlottenburg

Spandauer Damm 130

D-1000 Berlin 19

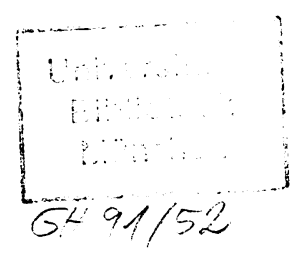

CIP-Titelaufnahme der Deutschen Bibliothek

Angiologische Notfälle /W. Hepp (Hrsg.). - Darmstadt: Steinkopff, 1990

ISBN 3-7985-0863-1

NE: Hepp, Wolfgang [Hrsg.]

Dieses Werk ist urheberrechtlich geschützt. Die dadurch begründeten Rechte, insbesondere die der Übersetzung, des Nachdrucks, des Vortrages, der Entnahme von Abbildungen und Tabellen, der Funksendung, der Mikroverfilmung oder der Vervielfältigung auf anderen Wegen und der Speicherung in Datenverarbeitungsanlagen, bleiben, auch bei nur auszugsweiser Verwertung, vorbehalten. Eine Vervielfältigung dieses Werkes oder von Teilen dieses Werkes ist auch im Einzelfall nur in den Grenzen der gesetzlichen Bestimmungen des Urheberrechtsgesetzes der Bundesrepublik Deutschland vom 9. September 1965 in der Fassung vom 24. Juni 1985 zulässig. Sie ist grundsätzlich vergütungspflichtig. Zuwiderhandlungen unterliegen den Strafbestimmungen des Urheberrechtsgesetzes.

Copyright (C) 1991 by Dr. Dietrich Steinkopff Verlag, GmbH \& Co. KG, Darmstadt Verlagsredaktion: Sabine Müller - Herstellung: Heinz J. Schäfer

\section{Printed in Germany}

Die Wiedergabe von Gebrauchsnamen, Handelsnamen, Warenbezeichnungen usw. in dieser Veröffentlichung berechtigt auch ohne besondere Kennzeichnung nicht zu der Annahme, daß solche Namen im Sinne der Warenzeichen- und Markenschutzgesetzgebung als frei zu betrachten wären und daher von jedermann benutzt werden dürften.

Gesamtherstellung: Brühlsche Universitätsdruckerei, Gießen Gedruckt auf säurefreiem Papier 


\section{Inhaltsverzeichnis}

Vorwort . . . . . . . . . . . . . . . . V V

Entwicklung der Gefäßchirurgie in West-Berlin

Rücker, G. . . . . . . . . . . . . . . . . . . . . . . . . . . . . . 1

Akuter Extremitäten-Arterienverschluß

Therapeutische Möglichkeiten der arteriellen Katheterthrombolyse beim akuten

Extremitätenarterienverschluß

Ranft, J. . . . . . . . . . . . . . . . . . . . . . . . . . 5

Der akute Extremitätenverschluß aus chirurgischer Sicht

Pallua, C., W. Hepp . . . . . . . . . . . . . . . . . . . . . . . . . 13

Die akute Ischämie im hohen Lebensalter

Kania, U., A. Dabelstein, L. C. Tung, R. Häring . . . . . . . . . . . . . 21

Die arterielle Embolie an der oberen Extremität (EOE): 254 Fälle

Kretz, J. G., D. Paneau, E. Weiss, F. Mantz, N. Chakfe, B. Mettauer,

R. Kieny .

Die Problematik des Poplitea-Aneurysmas

Schröder, A., H. Imig, R. Horstmann . . . . . . . . . . . . . . . . . . 35

Phlebothrombose der unteren Extremitäten

Die tiefe Venenthrombose der unteren Extremität aus angiologischer Sicht

Heidrich, H. . . . . . . . . . . . . . . . . . . . 43

Die Phlebothrombose der unteren Extremitäten aus chirurgischer Sicht

Stiegler, H., H. Arbogast, E. Hiller, G. Heberer . . . . . . . . . . . . . . 49

Aortendiagnostik

Bildgebende Verfahren in der Akutdiagnostik der thorakalen und abdominellen Aorta

Langer, M., R. Langer, J. Treisch, F. Astinet . . . . . . . . . . . . . . . 59

Gefäßverletzungen

Traumatische Gefäßverletzungen der Extremitäten

Hamann, H. . . . . . . . . . . . . . . . . . . . . . . . . . . . . 69

Iatrogene Gefäßverletzungen

Hepp, W., N. Pallua, B. Etmer . 


\title{
Die Phlebothrombose der unteren Extremitäten aus chirurgischer Sicht
}

\author{
H. Stiegler ${ }^{1}$, H. Arbogast ${ }^{1}$, E. Hiller ${ }^{2}$, G. Heberer ${ }^{1}$ \\ ${ }^{1}$ Chirurgische Klinik und Poliklinik der Universität München, \\ Klinikum Großhadern (Direktor: Prof. Dr. Dr. h.c. G. Heberer) \\ 2 3. Medizinische Klinik der Universität München, Klinikum Großhadern \\ (Direktor: Prof. Dr. W. Wilmanns)
}

\section{Einleitung}

Die tiefe Bein-Beckenvenenthrombose bedroht den Patienten akut durch eine Lungenembolie und beeinflußt dessen Lebensqualität langfristig durch das postthrombotische Syndrom. Auch wenn insbesondere durch eine konsequentere und verbesserte Thromboseprophylaxe die tiefe Venenthrombose seltener geworden ist, so wird der Chirurg dennoch mit diesem Krankheitsbild regelmäßig konfrontiert $(6,9)$. Zum einen ist er zur Therapie der Patienten aufgefordert, die auf der eigenen Station postoperativ eine tiefe Bein-Venenthrombose erleiden. Zum anderen wird er nicht selten zur Therapie einer tiefen Venenthrombose Stellung beziehen müssen, die spontan entstand und bei der allenfalls Risikofaktoren zu eruieren sind. Dabei erschwert insbesondere der individuelle Charakter der Erkrankung eine generelle Stellungnahme zur jeweils richtigen Therapieform, und es bedarf eines ganz gründlichen Abwägens zahlreicher Faktoren, um ein individuelles, maßgerechtes Therapiekonzept zu entwickeln.

An dieser Stelle sei es dem Chirurgen gestattet, zunächst seinen Beitrag der venösen Thrombektomie zu erläutern, zur Indikationsstellung wird später Stellung bezogen.

\section{Die venöse Thrombektomie}

Unter dem Schutz einer PTT-wirksamen systemischen Heparinisierung wird der Patient in Anti-Trendelenburg-Lagerung und Intubationsnarkose vorbereitet. Die Hautdesinfektion umfaßt das erkrankte Bein sowie Abdomen und Thorax, um bei einer intraoperativ möglichen Lungenembolie im Bedarfsfall auch thorakotomieren zu können. Auch ein Desinfizieren der kontralateralen Leiste ist ratsam. Über einen inguinalen Zugang werden V. femoralis comm., superf. und profunda freigelegt. Dabei folgt man der V.saphena magna als Leitschiene; die Gefäßdarstellung beschränkt sich auf wenige Zentimeter, um die Vene nicht unnötig aus dem Aufhängeapparat zu befreien.

Für den dann folgenden Thrombektomieakt der Beckenetage (Abb.1) ist vom Anästhesisten ein positiver endexspiratorischer Druck (PEEP) zu fordern, der erst dann ausreichend ist, wenn es durch Reduktion des preload zu einer im Blutdruck deutlich meßbaren Kreislaufdepression gekommen ist. Dies ist die entscheidende Maßnahme zur Verhinderung einer intraoperativen Lungenembolie. Nach Blut- 


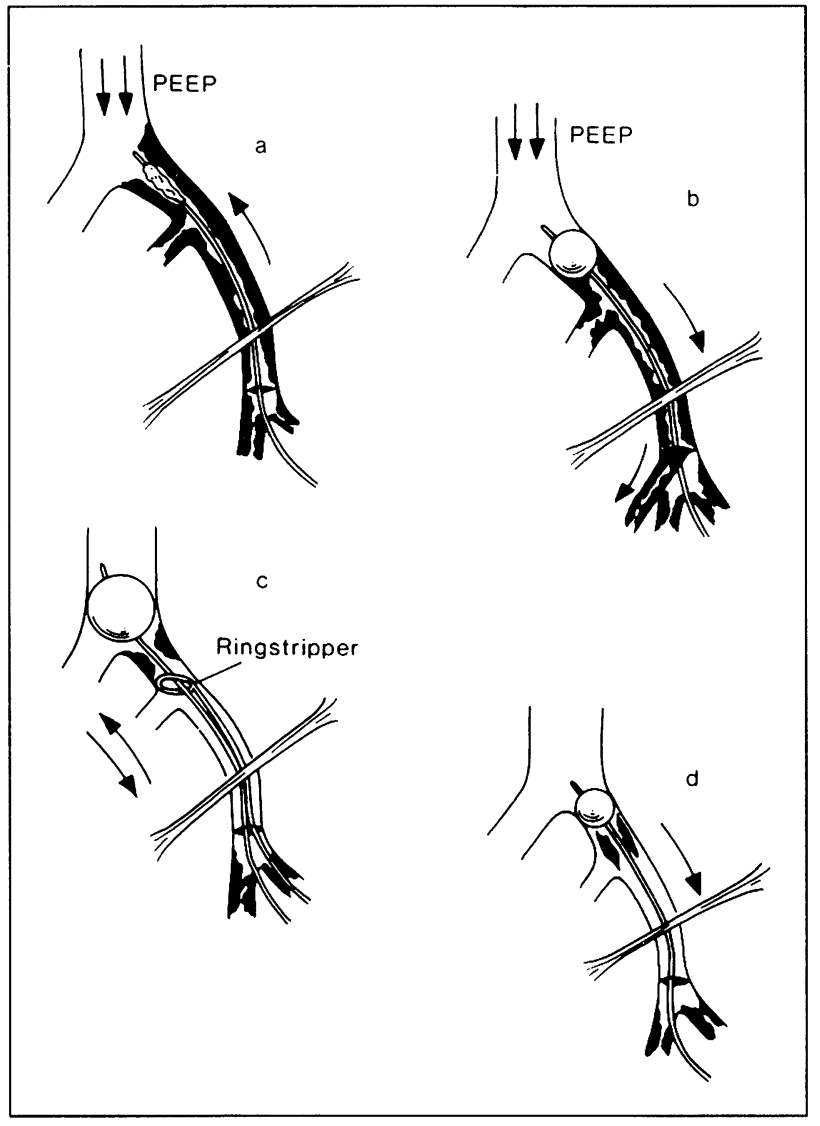

Abb. 1a-d. Thrombektomie der Beckenetage. a Unter hohem PEEP (s. Text) wird der FogartyKatheter in die V.cava inf. plaziert. b Mit der Retraktion des gefüllten Katheters wird das thrombotische Material geborgen. $\mathbf{c}$ Sind bei der intraoperativen Kontrolle thrombotische Residuen nachzuweisen, so wird der Fogarty-Katheter nochmals eingeführt und in infrarenaler Lage blockiert. Mit dem über den Katheter eingefädelten Ringstripper läßt sich dann das meist ältere wandständige Material lösen und $\mathbf{d}$ durch den Katheter entfernen

druckabfall wird die V. femoralis comm. quer eröffnet und der Fogarty-Katheter in die infrarenale V. cava inf. plaziert. Er wird gefüllt, mit wohldosiertem Druck zurückgezogen und der Thrombus so geborgen (Abb.1 b). Dieses Vorgehen wird wiederholt, bis kein thrombotisches Material mehr gewonnen werden kann.

Nach Abklemmen der V.femoralis comm. folgt dann die Thrombektomie des Beines. Hierzu wird die Extremität mit einer Esmarch-Binde ausgewickelt. Während der Finger des Assistenten die Venotomie leicht komprimiert, um den Blutverlust in Grenzen zu halten, treibt der Operateur durch kräftige Schläge gegen die Wade und den Oberschenkel das thrombotische Material aus. Auch diese Maßnahme ist mehrfach zu wiederholen. Da dies einen Blutverlust nach unserer Erfahrung von mindestens 11 zur Folge hat, sollte möglichst prinzipiell ein cell saver eingesetzt werden. 


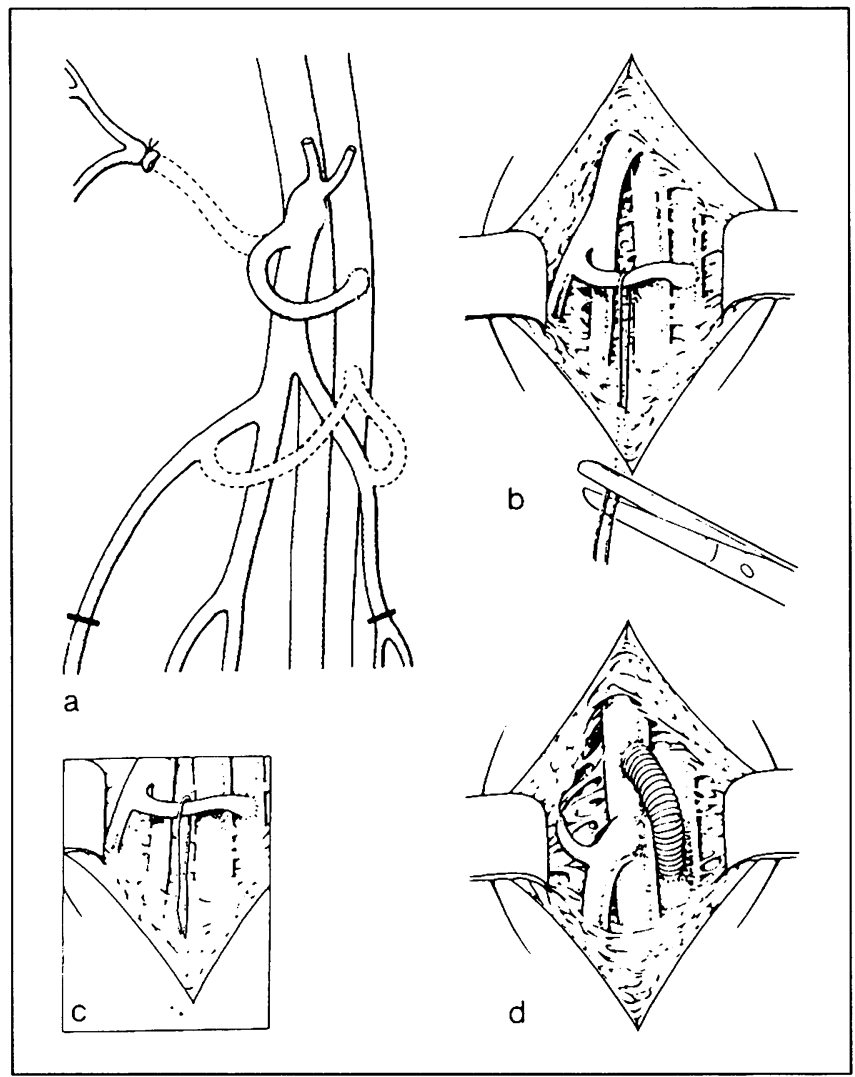

Abb. 2 a-d. Anlage einer inguinalen arteriovenösen Fistel. a Findet man bei der Präparation des Venensternes einen größeren Venenast, so wird dieser isoliert und zur A.femoralis superf. eingeschwenkt. Die Anastomose erfolgt dann in Korbhenkelform. b Zur Markierung der Fistel wird diese mit einem Blumendraht angeschlungen, der distal des unteren Wundwinkels ausgeleitet wird. c Nach Abschneiden des Drahtes gleiten die Enden in das subkutane Fettgewebe zurück, sie dienen als Leitschiene zum Wiederauffinden der Fistel. d Liegt kein ausreichend großkalibriger Venenast vor oder ist dieser bei ungünstigem Winkel nach dem Einschwenken geknickt (Frühverschluß!), so steht alternativ auch ein Goretex-Implantat ( $\varnothing 5 \mathrm{~mm}$, expanded ohne Ringverstärkung) zur Verfügung. Dieses wird zwischen A. femoralis superf. und V. femoralis comm. anastomosiert. Die Markierung erfolgt wie in $\mathbf{b}$ und $\mathbf{c}$

Nach Beendigung der Thrombektomie folgt die Phlebographie der Beckenetage. Mit dieser wird die Vollständigkeit der Thrombektomie überprüft. Neuerdings führen wir auch die von Vollmar favorisierte Phleboskopie durch, da bei der phlebographischen Gefäßbeurteilung Fehlinterpretationen aufgrund von Überlagerungen des kontrastierten Gefäßes mit Wirbelsäule und Becken nicht zu vermeiden sind. Sind thrombotische Residuen zu erkennen - und diese sind häufig in der Spornregion lokalisiert - so wird der erneut eingeführte Fogarty-Katheter in infrarenaler Position geblockt, über diesen der Ringstripper eingefädelt und das wandständige Material gelöst (Abb. 1 c). Nach Entfernen des Ringstrippers wird dann der Fogarty-Katheter 
zurückgezogen und das ältere thrombotische Material entfernt. Bereits hier muß jedoch betont werden, daß diese Maßnahmen der Thrombektomie nur gelingen, wenn das thrombotische Material frisch und noch keine wesentliche Organisation und somit Fixation des Thrombus an der venösen Wand erfolgt ist $(2,12,14,15)$.

Zur Frage nach der Indikation einer passageren arteriovenösen Fistel beziehen wir folgende Stellung: Die Implantation einer Fistel ist dann indiziert, wenn das Rethromboserisiko erhöht ist:

1. beim Belassen von thrombotischem Material in der Beckenetage,

2. bei der Anwendung des Ringstrippers (stärkerer Endothelschaden),

3. bei Thrombektomie von altem thrombotischen Material.

Die av-Fistel verbleibt für 6 Monate in inguinaler Position und wird dann verschlossen (Abb. 2).

Während dieses Vorgehen bis auf wenige Modifikationen standardisiert ist, bleibt die Frage nach wie vor aktuell, welches denn in der jeweiligen Situation die richtige Therapieform ist: Operation, Heparin oder Lyse.

\section{Indikationsstellung: Operation, Heparin oder Lyse?}

Sucht man nach einer Antwort auf diese Frage in der Literatur, so wird man enttäuscht. Die meisten Therapiestudien sind retrospektiv angelegt und berücksichtigen in der Regel nur zwei Therapieformen. Außerdem werden uneinheitliche Bewertungsmaßstäbe für die Ergebnisse zugrundegelegt und zudem funktionelle Aspekte wenig berücksichtigt $(1,3-5,7,11,12)$. Wir haben deshalb in einer prospektiven Studie vom 1.2.1984 bis zum 1.7.1986 alle im Klinikum Großhadern diagnostizierten tiefen Venenthrombosen erfaßt und deren Therapie nach interdisziplinärem Kontakt festgelegt - eine Randomisierung wurde aus ethischen Gründen nach eingehender Diskussion abgelehnt. Als Therapieformen wählten wir:

1. Operation wie oben beschrieben. Re-Thromboseprophylaxe mit PTT-wirksamer Heparinisierung (80-120 s), später Markumarisierung für 6 Monate, Kompressionsbehandlung.

2. Heparin: 10000 I.E. als Bolus i.v., dann PTT (80-120 s)-gesteuert über Perfusor. Überlappende Markumarisierung für 6 Monate, Kompressionsbehandlung.

3. Lyse mit Streptokinase bzw. Urokinase $(250000 \mathrm{E}$ in $20 \mathrm{~min}$ i.v., dann $100000 \mathrm{E}$ pro h. Später Markumarisierung für 6 Monate, Kompressionsbehandlung (8).

In die Studie wurden 209 Patienten mit 233 tiefen Venenthrombosen aufgenommen. Davon wurden $29 \%$ operiert $(n=68), 53 \%$ heparinisiert $(n=124)$ und $18 \%$ lysiert $(n=41)$. Die Altersverteilung ist aus Abb. 3 zu entnehmen. Hier geht unter anderem die Prämisse ein, daß Patienten mit einem Alter über $65 \mathrm{~J}$. in der Regel ein schweres postthrombotisches Syndrom nicht mehr erleiden und deshalb nur in Ausnahmefällen operiert oder lysiert werden sollten.

Beim Abwägen, welcher Therapieform der Vorzug zu geben ist, waren zahlreiche Kontraindikationen zu beachten. Diese sind in den Abb. 4 und 5 festgehalten. So überwiegt bei den Kontraindikationen zur Operation die Thrombuslokalisation $(22 \%)$. Hierunter sind alle Patienten mit Unterschenkelthrombose einzuordnen, da diese mit dem indirekten Verfahren der Thrombektomie schwer erreichbar sind und zudem eine hohe Spontanlyserate aufgrund eines günstigen Verhältnisses von Endotheloberfläche zu Thrombusmasse aufweisen. Ein Thrombusalter über 14 Tage 


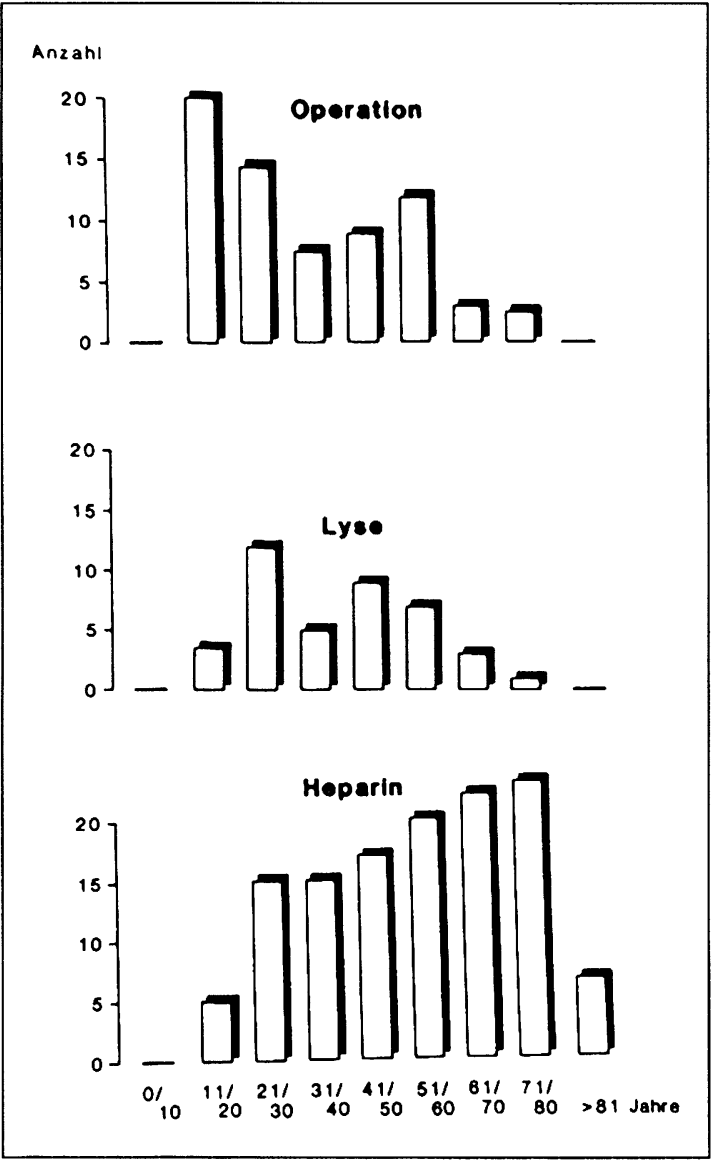

Abb. 3. Altersverteilung der Patienten mit tiefer Bein-Beckenvenenthrombose (erfaßt sind nur die Patienten, die in die prospektive Studie aufgenommen wurden; tiefe Venenthrombosen, $n=233$, Zeitraum 1.2.1984 bis 1.7.1986)

(subtile Anamnese, bei zweizeitigem Thrombuswachstum zählt die Symptomatik des letzten Thrombuswachstums) war in $21 \%$ eine Kontraindikation. Ebenso war ein nicht beherrschbares Tumorleiden eine Kontraindikation für eine der beiden aggressiven Therapieformen. Die begleitende Lungenembolie war in $9 \%$ Grund, gegen die Operation und für die Lyse zu entscheiden, um sowohl die Emboliequelle als auch das Emboliezielorgan zu behandeln.

Häufigste Kontraindikation für eine Lyse war eine vorausgegangene Operation (innerhalb 10 Tage postop.), die Unterschenkelvenenthrombose wurde in der Regel auch nicht lysiert.

Um bei der Beurteilung der Ergebnisse sowohl klinisch-anamnestische, morphologische (Phlebographie) als auch funktionelle Aspekte zu berücksichtigen, wurde der in Abb. 6 gezeigte Score entwickelt. Ich betone besonders, daß die Miteinbeziehung funktioneller Parameter wichtig ist, da diese letztlich über das hämodynamische Verhalten Aufschluß geben. Als funktionelle Meßmethode wählten wir die Phlebodynamometrie (10). 


\section{Thrombuslokalisation}

\section{Thrombusalter}

\section{Tumorleiden}
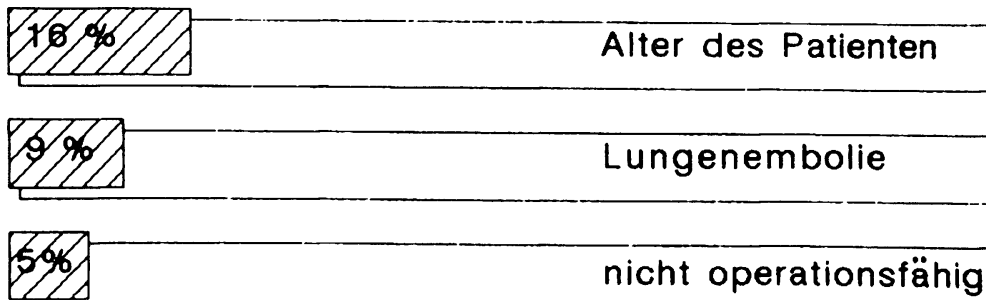

Abb.4. Kontraindikationen zur Operation

Legt man nun diesen Score den Nachuntersuchungen 6 Monate nach Therapie zugrunde, so erhält man die in Abb. 7 gezeigten Ergebnisse. Lyse und Operation führen zu nahezu gleich guten Therapieresultaten. Aber auch in der Heparingruppe finden sich erstaunlich gute Ergebnisse. Bei differenzierter Betrachtung gewinnt man jedoch ein ganz anderes Bild. So sind die Drei-Etagen-Thrombosen in der Gruppe mit den jeweils sehr guten Ergebnissen bei der Operation mit $45 \%$, bei der Lyse mit $8,3 \%$ und in der Heparingruppe mit $0 \%$ (!) vertreten. Nur die partiellen Unterschenkelvenenthrombosen waren mit Heparin sehr gut zu behandeln. Somit sind die Kollektive nur bedingt vergleichbar, da in der Operationsgruppe eine Selektion zum schwereren Krankengut erfolgte.

Bildet man eine Untergruppe mit der Einschränkung: Venenthrombose der drei Etagen und Alter bis zu 8 Tagen, so bringt die Operation klar die besseren Ergebnisse (Abb. 8). Hier muß allerdings eingeschränkt werden, daß bei einer Gruppenbildung dieses so heterogenen Krankengutes naturgemäß letztlich kleine Kollektive resultieren.

Während somit Heparin zu Lyse und Operation beim Zwei- und DreiEtagenbefall keine Alternative ist, bleibt noch die Frage zu klären, ob Lyse und Operation im klinischen Alltag konkurrieren. Diese Frage ist klar mit nein zu beantworten. Denn nach Berücksichtigung aller Kontraindikationen verbleiben nur knapp 17\% der Patienten, bei denen beide Therapieformen alternativ möglich gewesen wären (13).

Die Ergebnisse dieses kleinen Kollektivs 6 Monate nach Behandlung zeigt Abb.9. Hier finden sich ebenfalls kaum Unterschiede beim Vergleich beider Therapieformen. 


\begin{tabular}{|l|}
\hline \\
\hline
\end{tabular}

Abb. 5. Kontraindikationen zur Lyse

\begin{tabular}{|c|c|}
\hline sehr gut & $\begin{array}{l}\text { Keine Beschwerden } \\
\text { Phlebographie: frei } \\
\Delta p>50 \mathrm{~mm} \mathrm{Hg}\end{array}$ \\
\hline gut & $\begin{array}{l}\text { keine Beschwerden } \\
\text { Phlebographie: geringgradige Residuen } \\
\Delta p>50 \mathrm{~mm} \mathrm{Hg}\end{array}$ \\
\hline ausreichend & $\begin{array}{l}\text { Beschwerden abends } \\
\text { Phlebographie: AbfluB behindert } \\
\triangle p=35-50 \mathrm{~mm} \mathrm{Hg}\end{array}$ \\
\hline schlecht & $\begin{array}{l}\text { Beschwerden ganztägig } \\
\text { Phlebographie: AbfluB behindert } \\
\triangle p<35 \mathrm{~mm} \mathrm{Hg}\end{array}$ \\
\hline
\end{tabular}

Abb.6. Einteilungskriterien für die Beurteilung der Ergebnisse nach Therapie tiefer BeinBeckenvenenthrombosen 


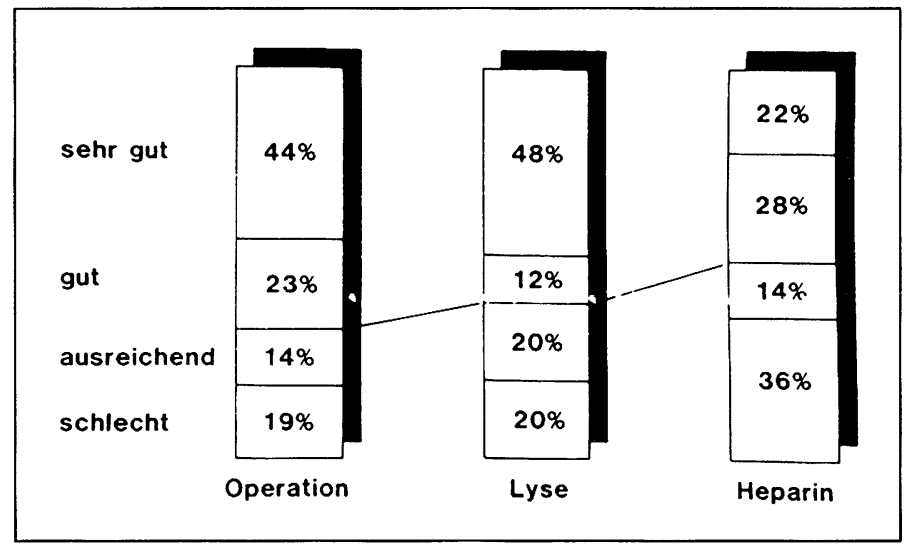

Abb. 7. Ergebnisvergleich nach Therapie tiefer Bein-Beckenvenenthrombosen (summarisch)

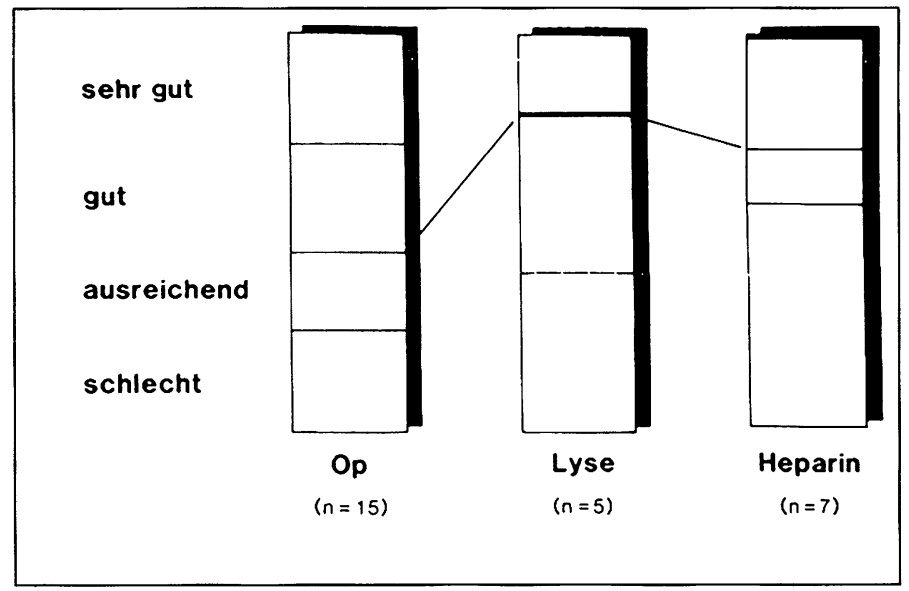

Abb. 8. Ergebnisvergleich der drei Therapieformen bei einem Thrombosealter bis zu 8 Tagen und beim Drei-Etagenbefall

Aus chirurgischer Sicht und - da unsere Daten in guter Zusammenarbeit mit unseren Angiologen erstellt wurden - auch aus der Sicht unserer Angiologen befürworten wir folgende Indikationen:

1. Eine Venenthrombose (VT) über 14 Tage wird heparinisiert.

2. Alle Kontraindikationen (s. Abb. 4 und 5) sind zu beachten.

3. Unterschenkel-VT werden in aller Regel heparinisiert.

4. Oberschenkel-Unterschenkel-VT werden bevorzugt lysiert.

5. Beim Drei-Etagenbefall wird die Operation favorisiert.

6. Nach einer ineffektiven Lyse muß die Operationsindikation nochmals überprüft werden (der durch die Lyse veränderte Thrombus läßt sich in der Regel besonders leicht entfernen). 


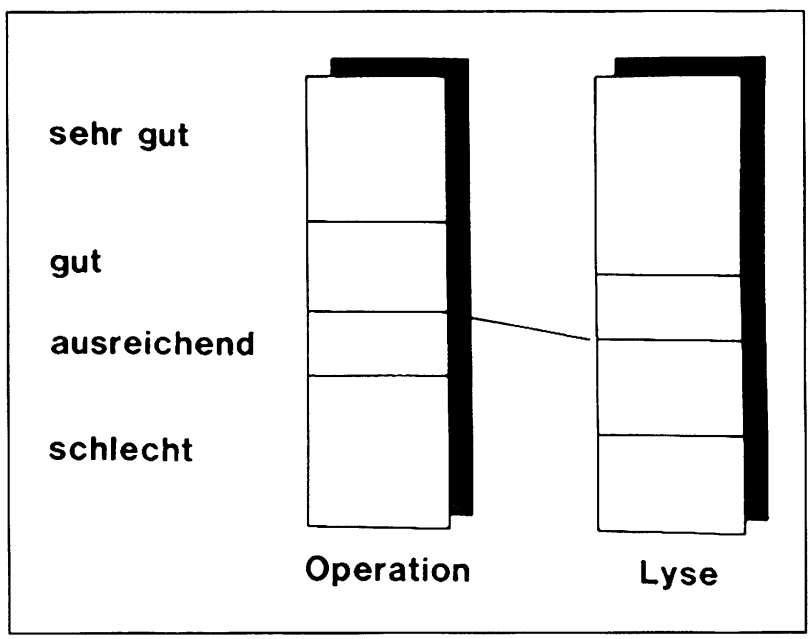

Abb. 9. Ergebnisvergleich nach Operation $(n=20)$ bzw. Lyse $(n=19)$ bei fehlenden Kontraindikationen

Für ein gutes Therapieergebnis ist eine nachfolgende konsequente Markumarisierung und Kompressionsbehandlung über 6 Monate notwendig.

Aus dem bisher Geschilderten wird deutlich, daß jede tiefe Venenthrombose ihre eigene individuelle Vorgeschichte und Problematik hat. Es bedarf daher einer guten interdisziplinären Zusammenarbeit, um in der aktuellen Situation die richtigen Entscheidungen zu treffen.

\section{Literatur}

1. Arnesen H, Hoiseth A, Ly B (1982) Streptokinase or heparin in the treatment of deep venous thrombosis - Follow-up results of a prospective study. Acta Med Scand 211:65

2. Baumann G (1974) Operative Technik bei der akuten Bein-Beckenvenenthrombose unter Berücksichtigung von Venenverletzungen. Vasa 3:34

3. Bieger R, Boekhout-Mussert RJ, Hohmann F, Loeliger EA (1976) Is streptokinase useful in the treatment of deep vein thrombosis? Act med scand 199:81

4. Brunner U, With W (1971) Spätresultate nach Thrombektomie bei lliofemoralvenenthrombose im klinisch-radiologischen Vergleich. Schweiz med Wschr 101:1327

5. Common HH, Seaman AJ, Rösch J, Porter JM, Dotter CT (1976) Deep venous thrombosis treated with streptokinase or heparin. Follow-up of a randomized study. Angiology 27:645

6. Encke A, Breddin K, Biegholt M, Stiegler H et al. (1988) Comparison of a low molecular weight heparin and unfractionated heparin for the prevention of deep vein thrombosis in patients undergoing abdominal surgery. Br J Surg 75:1058

7. Fasolini FG, Streuli HK (1985) Thrombektomie versus konservative Therapie tiefer BeckenBein-Venenthrombosen. Spätergebnisse 10 Jahre danach. Helv Chir Acta 52:735

8. Hiller E, Riess H (1988) Hämorrhagische Diathese und Thrombose. Wiss Verl-Ges, Stuttgart

9. Kakkar VV, Djazaeri B, Fok J, Fletcher M, Scully MF, Westwick J (1982) Low-molecularweight heparin and prevention of postoperative deep vein thrombosis. Br Med J 284:375

10. Kriessmann A, May R (1978) Empfehlungen zur Standardisierung und praktischen Anwendung der peripheren Venendruckmessung. In: May R, Kriessmann A (Hrsg) Periphere Venendruckmessung. Thieme, Stuttgart 
11. Minar E, Ehringer H, Marosi L, Piza F, Wagner O, Czembirek H (1983) Klinische, funktionelle und morphologische Spätergebnisse nach venöser Thrombektomie. Vasa 12:346

12. Raithel D, Söhnlein B (1981) Die venöse Thrombektomie - Technik und Ergebnisse. Vasa 10:119

13. Stiegler H, Arbogast H, Nees S, Halder A, Grau A, Riess H (1989) Thrombectomy, lysis or heparin treatment: concurrent therapies of deep vein thrombosis: therapy and experimental studies. Sem Throm Hem 15:250

14. Stiegler H, Sunder-Plassmann L, Becker HM (1985) Indikationen und Technik bei BeckenBeinvenenthrombosen. Chirurg 56:73

15. Stiegler H, Sunder-Plassmann L (1987) Akute Verschlüsse der Venen. In: Heberer G, van Dongen RJAM (Hrsg) Gefäßchirurgie. Springer, Berlin

Anschrift des Verfassers:

PD Dr. H. Stiegler

Chirurgische Klinik und Poliklinik

der Universität München

Klinikum Großhadern

D-8000 München 